\title{
Laparoscopic Surgery for Renal Cell Carcinoma
}

\author{
Department of Urology Nagoya University Graduate School of Medicine, Nagoya, Japan \\ Yoshinari Ono
}

Laparoscopic surgery for renal cell carcinoma consists of both radical and partial nephrectomy. Laparoscopic radical nephrectomy was performed by us and Clayman et al. in 1992, and has been done since in over 10,000 patients with renal cell carcinoma worldwide by Barrett et al, Rassweiler et al, Abbou et al, Kavoussi et al, Janetschek et al, and Gill et al. In the initial period the operative time was often over 5 hours, but the estimated blood loss was less than $300 \mathrm{ml}$. Patients could have meals and walk on the first postoperative day with full convalescence achieved within 3-4 weeks after surgery. Laparoscopy seems to have a minimally invasive nature. Today, the operative time is $3-4$ hours, and the estimated blood loss is less than $200 \mathrm{ml}$. As to long-term cancer control, the 5 -year survival rate was $90-95 \%$ in patients with pT1N0/X disease, $75 \%$ in our patients with pT2N0 disease and $100 \%$ in our patients with pT3aN0 disease. In patients with pT3b disease, Gill et al and we successfully removed kidneys with tumor thrombi in the renal vein. The long-term cancer controls of laparoscopic radical nephrectomy are comparable to those of open surgery for patients with even locally advanced disease.

Laparoscopic partial nephrectomy has technical difficulties in the diagnosis of the location of the tumor mass, the control of bleeding from the incised renal parenchyma, and closing the injured renal calyx and pelvis. We could not perform the technique in clinical practice before developing new devices for incision and coagulation. In 1992, Winfield, McDougall, Kavoussi, and Clayman applied the procedure without vascular clamps while using electrosurgical scissors and an argon beam coagulator. They successfully treated various diseases including renal cell carcinoma. Janetschek et al. performed the technique without vascular clamps using a laser and an argon beam coagulator for removing small renal mass in 7 patients between 1994 and 1996. In 1998, Gill et al applied laparoscopic Satinsky clamping of the renal artery and vein during partial nephrectomy. They described their operative outcomes of 200 patients treated with laparoscopy, in which they used vascular clamps to control bleeding during dissection of the tumor mass. Mean operative time was 2.8 hours, and mean estimated blood loss was $270 \mathrm{ml}$. The $12 \%$ complication rate included prolonged urinary leakage and bleeding. Janetschek et al also described their operative outcome of 25 patients treated with laparoscopic partial nephrectomy without using a vascular clamp during dissection of the tumor mass. Mean operative time was 2.7 hours, and mean estimated blood loss was $287 \mathrm{ml}$. The complication rate was $12 \%$. There is no definitive data of long-term cancer control or renal function after laparoscopic partial nephrectomy. Technical consensus is needed for how to diagnose the tumor mass and how to repair the incised renal parenchyma and/or renal calices and pelvis. Much more data on cancer control is needed. Present data indicates only that laparoscopic partial nephrectomy is also available for treating the patient with small renal cell carcinoma. 